\title{
eRock: An Open-Access Repository of Virtual Outcrops for Geoscience Education
}

\author{
Adam J. Cawood', Clare E. Bond, Dept. of Geology \& Petroleum Geology, University of Aberdeen, AB24 $3 U E$, UK
}

Visualizing objects and structures in $3 \mathrm{D}$ is a task that most people, including geologists, find difficult. Geoscience educators and students often find fieldbased exercises the most effective medium for understanding complex geological concepts and visualizing relationships in 3D (e.g., Elkins and Elkins, 2007). Unfortunately, field-based training is not available to everyone. Traditional barriers to this field-based training include physical disabilities, lack of financial resources, and geographical restrictions. Recent advances in data acquisition and processing have the potential to circumvent these traditional barriers to access and open up a vast number of field sites to a diverse range of people.

\section{D RECONSTRUCTIONS FOR RESEARCH AND COMMUNICATION}

Advances in computer technology and increased availability of affordable acquisition tools mean that $3 \mathrm{D}$ reconstructions of outcrop and landscapes are increasingly used by the geoscience community as a research tool. Virtual outcrops derived from LiDAR and digital photogrammetry can provide a wealth of detailed geological information (e.g., Cawood et al., 2017) and as such, these $3 \mathrm{D}$ realizations are gaining traction as an accessible means of communicating geoscience within industry and academia. In spite of these developments, little has been done to leverage this technology as a tool for education in the geosciences. We attribute this to (1) the requirement, until recently, for specialist software packages to render 3D digital objects, and (2) the widespread use of inaccessible, proprietary data banks to store virtual outcrops used in applied research.

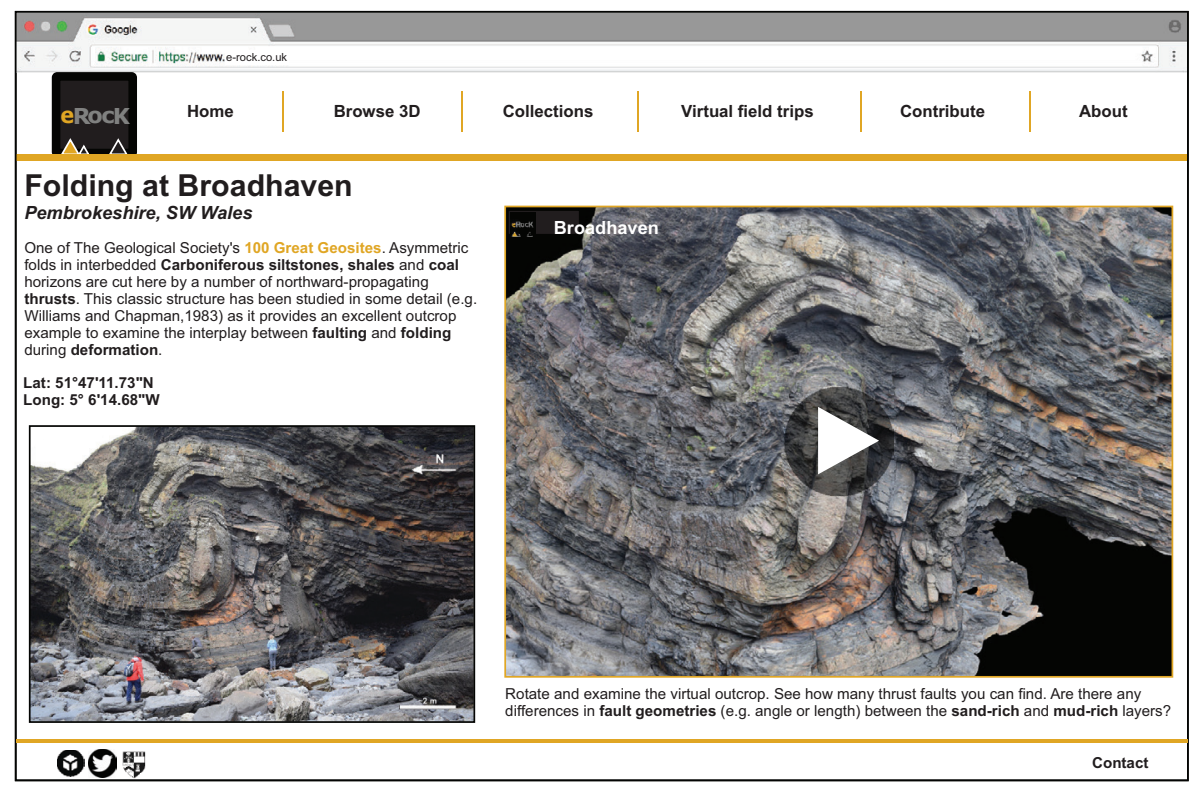

Figure 1. Example of a virtual outcrop page at eRock (www.e-rock.co.uk/broadhaven).

\section{PUBLIC-DOMAIN DIGITAL \\ ARCHIVES: FROM 2D TO 3D}

The past two decades have seen a surge in the number of open-access digital archives available online, such as curated collections of public-domain art (e.g., www.metmuseum.org). Sculptures and objects in these digital collections are typically shown in pseudo-3D by capturing them through a series of images from different viewpoints. Recent advances in $3 \mathrm{D}$ rendering, however, mean that these objects can now be digitally reconstructed and made available online in true 3D format through online viewers. Sketchfab (www.sketchfab.com), a resource originally made for the gaming and computer animation community, is the best known of these viewers.

Sketchfab is being used by a growing number of educators, scientists, and archivists: examples include 3D anatomical models for e-learning in medical sciences, digitally reconstructed archaeological sites, and digital versions of culturally important objects (e.g., The Rosetta Stone; www.sketchfab.com/britishmuseum). This variety of content suggests that $3 \mathrm{D}$ rendering has great potential as a tool in a wide variety of applications, from digitally preserving cultural heritage to helping students understand the 3D structure of objects. Currently, however, this resource has not yet been systematically developed in the geoscience community - there remains a lack of efficiently collated, open-access 3D material online.

\section{eROCK: AN OPEN-ACCESS REPOSITORY OF VIRTUAL OUTCROPS}

eRock (www.e-rock.co.uk) is an online resource that presents virtual outcrops through an accessible, geoscience-focused

GSA Today, https://doi.org/10.1130/GSATG373GW.1. Copyright 2018, The Geological Society of America. CC-BY-NC.

${ }^{1}$ Email: adam.cawood@abdn.ac.uk 
platform (Fig. 1). By embedding

Sketchfab-hosted models directly into eRock, curated collections can be presented in a focused, easy-to-follow format: Users can view virtual outcrops without the need for searching through large amounts of irrelevant material, with little associated data or context. 3D models can currently be browsed in eRock by location (through a map interface), by theme (e.g., metamorphic or sedimentary), or by virtual field trip (e.g., the geology of the NW Highlands, Scotland). Concise outcrop descriptions are provided with key information, links to references, metadata, and other relevant resources for each 3D model (Fig. 1). All eRock models are open access, free to download, and 3D viewable through a standard web browser, with no need for specialist software packages.

Current demographic data for the site show that the largest user group is the 35-44 age group, followed by 25-34-yearolds. The $18-24$ age group currently only accounts for $11 \%$ of visitors (Fig. 2A) but spends the longest average time at the site per visit (Fig. 2B), suggesting that they visit multiple pages and virtual outcrops per session. Unfortunately, both women and those from low- and middle-income countries are underrepresented as a proportion of total visitors to the site (Fig. 2). As the aim of the project is to build eRock into a collaborative educational tool for users across the spectrum of educational background and needs, our future efforts need to be directed at widening the range and diversity of people who visit the site.

\section{VIRTUAL OUTCROPS FOR EDUCATION}

Field-based exercises have been shown to enhance geological understanding. We suggest that digital realizations of outcrops may improve visualization and understanding in much the same way, but only if virtual outcrops are provided in context. Unstructured collections of data devoid of geological description or linked reference material are unlikely to provide much benefit. 3D visualizations must be provided with appropriate material to contextualize the object - in this way, students can understand the scale of the object they are looking at, its geological significance, and how it relates to the surrounding landscape

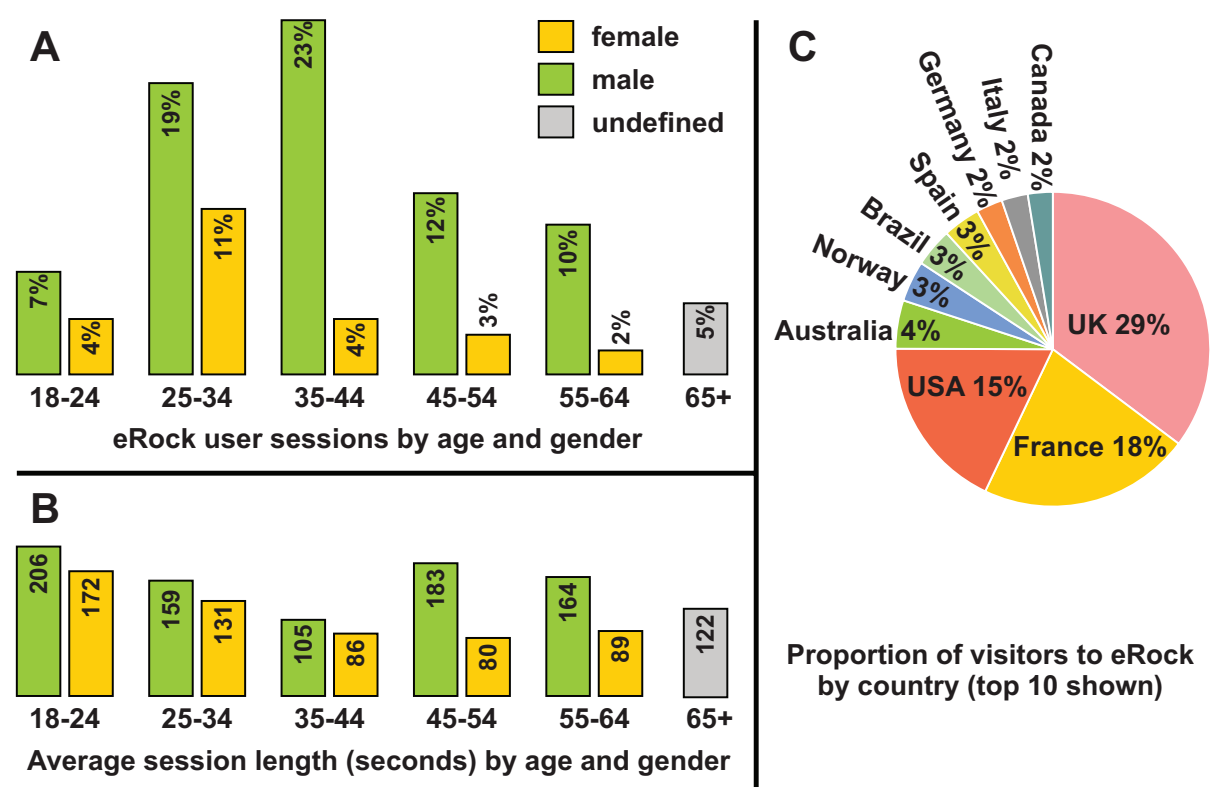

Figure 2. User demographic data for www.e-rock.co.uk.

and subsurface. The strength of eRock lies in its integrated approach: Many models are provided as part of a suite of educational materials, including geological maps, cross sections, field photographs, and text.

At the time of this writing, preliminary studies by the authors suggest that undergraduate students find a combination of traditional fieldwork and digital visualization useful: Students report an improvement in both their perception of 3D landscapes and their visualization of complex geological structures compared to fieldbased studies alone. Further, students appear to be strongly in favor of the use of virtual outcrops as part of the undergraduate geoscience curriculum.

\section{LOOKING FORWARD: \\ FUTURE OBJECTIVES}

1. eRock is in the early stages of development, with roughly 40 virtual outcrops available online. We encourage others to help the project grow by providing 3D models, images, site descriptions, and metadata. Structured, contextualized content is critical to ensure eRock is a useful tool-we need help from the wider community to achieve this.

2. It is important to represent a wide variety of outcrops, both in terms of location and geological theme. We want a diverse range of people to use this resourcea repository that showcases outcrops from around the world is more likely to attract visitors from a variety of countries and backgrounds.

3. We are currently running a series of trials that assess the relative merits of virtual outcrops versus traditional teaching materials and field-based exercises. If this approach genuinely improves students' geological understanding, the next stage will be to incorporate the technology into structured curricula. The success of this project depends on collaboration. Get in touch, and give us your input to help the project grow.

\section{REFERENCES CITED}

Cawood, A.J., Bond, C.E., Howell, J.A., Butler, R.W., and Totake, Y., 2017, LiDAR, UAV or compass-clinometer? Accuracy, coverage and the effects on structural models: Journal of Structural Geology, v. 98, p. 67-82, https:// doi.org/10.1016/j.jsg.2017.04.004.

Elkins, J.T., and Elkins, N.M., 2007, Teaching geology in the field: Significant geoscience concept gains in entirely field-based introductory geology courses: Journal of Geoscience Education, v. 55, no. 2, p. 126-132, https:// doi.org/10.5408/1089-9995-55.2.126.

ManusCript ReCEIVEd 3 Apr. 2018 REVISED MANUSCRIPT RECEIVED 31 OCT. 2018 MANusCRipt ACCEPTED 10 Nov. 2018 